Loewenstein, J., \& Gentner, D. (2001). Spatial mapping in preschoolers: Close comparisons facilitate far mappings. Journal of Cognition and Development, 2(2), 189-219.

\title{
Spatial Mapping in Preschoolers: Close Comparisons Facilitate Far Mappings
}

\author{
Jeffrey Loewenstein and Dedre Gentner \\ Department of Psychology \\ Northwestern University
}

\begin{abstract}
To test the hypothesis that comparison processes facilitate schema extraction, we studied the effect of making comparisons on 3-year-olds' ability to perform mapping tasks. In 3 studies, children were tested on their ability to find a hidden toy in a model room after being shown its location in a perceptually different room. In Experiment 1 we found that seeing 2 similar hiding events--permitting a sequential comparisonimproved 3-year-olds' performance on the mapping task. Experiment 2 showed a more striking effect: Simply comparing the initial hiding model with another nearly identical model helped children to succeed on the subsequent mapping task. Experiment 3 showed that the comparison effect was not simply due to an opportunity to interact with 2 examples, but was specific to comparing them. We conclude that comparing examples can facilitate children's noticing common relational schemasin this case, a spatial relational schema-and their ability to use this system of relations in subsequent tasks. Our central hypothesis is that the process of comparison is a major force in children's learning and development. In this work, we test the specific claim that drawing comparisons among similar spatial arrays fosters insight into the common spatial relations, as assessed in a subsequent spatial mapping task.
\end{abstract}

We tested the role of comparison in promoting relational insight by using a version of the classic spatial mapping task developed by DeLoache and her colleagues (DeLoache, 1987, 1989a, 1989b, 1990, 1995; DeLoache, Kolstad, \& Anderson, 1991; DeLoache, Miller, \& Rosengren, 1997; Uttal, Schreiber, \& DeLoache, 1995). However, whereas prior research with this task has focused chiefly on test-

Requests for reprints should be sent to Jeffrey Loewenstein, Department of Psychology, Northwestern University, 2029 Sheridan Road, Evanston, IL 60208-2710. E-mail: loewenstein@nwu.edu 
ing children's appreciation of symbol-referent relations, we focused on similarity relations. In the mapping task, children watched as a toy was hidden in one space, and then they were asked to find a toy hidden in the same place in a second space. This task requires children to make correspondences between the two spaces. As noted previously, there has been abundant research investigating the development of children's understanding of symbol-referent relations: that a model stands for a room (e.g., Bluestein \& Acredolo, 1979; DeLoache, 1987, 1995; DeLoache et al., 1997; Dow \& Pick, 1992; Liben \& Downs, 1989). An equally important aspect of model use is understanding in what respects the model matches the room: how the model is similar to the room (e.g., Blades \& Cooke, 1994; Blades \& Spencer, 1994; DeLoache et al., 1991; Marzolf, DeLoache, \& Kolstad, 1999).

Similarity and symbol-referent relations are distinct notions (Lillard, 1993; Perner, 1991). For instance, the Italian flag stands for, but is not similar to, the country of Italy, and yet the Italian flag is similar to, but does not stand for, the Irish flag. Models and maps are interesting composites. The items and their relative positions in a model both represent and are similar to objects and locations in the world. Our interest is in how children come to trace correspondences between two spaces.

Gentner and Rattermann (1991) focused on children's perception of similarities in the model task and suggested that processes of structural alignment and mapping come into play when children connect two spaces and draw inferences between them (Gentner 1983, 1989; Markman \& Gentner, 1993; Medin, Goldstone, $\&$ Gentner, 1993). According to structure-mapping theory, the comparison process entails aligning two structured representations. Each structured representation has elements that stand for objects and elements that stand for relations holding among those objects. Structure-mapping theory distinguishes between mappings based on object similarities-for example, chair $1 \rightarrow$ chair 2-from mappings based on relational similarities-for example, UNDER (toy 1, bed l) $\rightarrow$ $U N D E R$ (toy 2, bed 2). A related distinction between element correspondences and relational correspondences has been drawn in the spatial literature (e.g., Bluestein \& Acredolo, 1979). Liben (1999; Liben \& Yekel, 1996) described a comparable distinction between representational correspondences (e.g., a picture of a house on a map looks like a real house) and geometric correspondences (e.g., a picture of a house is in the same position relative to other items on the map as the real house is to its surroundings).

Both object similarity and relational similarity can promote mapping. An example of object similarity facilitating mapping comes from DeLoache et al. (1991), who showed that preschoolers performed better when the component objects in two spaces were similar to each other than when they were dissimilar. An example of relational similarity facilitating mapping comes from Marzolf et al. (1999). They showed that preschoolers performed better when the component objects in two spaces were placed in the same arrangement rather than in different arrangements. 
Although young children could potentially use object and relational similarities equally to perform mapping tasks, there is evidence that they rely primarily on object similarities (Bence \& Presson, 1997; Blades \& Cooke, 1994; Gentner \& Rattermann, 1991; Marzolf \& DeLoache, 1997; Rattermann \& Gentner, 1998a, 1998b, 2001). For example, Blades and Cooke (1994) asked children to map between two models containing four items of furniture, including two identical items (twins; i.e., $A B C_{1} C_{2} \rightarrow A^{\prime} B^{\prime} C_{1}{ }^{\prime} C_{2}{ }^{\prime}$ ). They reasoned that if children were mapping solely on the basis of matching objects, then they should search accurately for items with unique matches $\left(A \rightarrow A^{\prime}\right.$ and $\left.B \rightarrow B^{\prime}\right)$; however, they should choose at random for the two twin items, which have two potential object correspondences $\left(\mathrm{C}_{1} \rightarrow \mathrm{C}_{1}\right.$ ' or $\left.\mathrm{C}_{2}{ }^{\prime}\right)$. In contrast, if children are also able to align spatial relations, then they should be accurate with both the unique and twin items because they can use relative spatial position to disambiguate the twin items. Blades and Cooke found that 31-through 39-month-old children succeeded on the unique items but performed at chance on the twin items, indicating they carried out the mapping task on the basis of object matches. By 45 to 58 months of age, children performed well on the twin items, indicating that they were able to use relational correspondences.

A further demonstration of early object-focused mapping in the model task comes from studies by Marzolf and DeLoache (1997). Identical white boxes were placed on the furniture in a model, and larger white boxes were similarly placed in a matching room. Children at 38 months who succeeded on the normal modelroom task - that is, could accurately find a hidden toy placed under an item of furniture-failed to find the toy when it was hidden in a white box placed at an item of furniture. When children saw the toy hidden in a box in the model, they searched the boxes in the room, but they did not know which box to choose. It appears that they could perceive the object matches between the spaces (i.e., that the small white boxes matched the big white boxes), but they were unable to use the relational correspondences (i.e., that the small white box on the small couch matched the big white box on the big couch) to disambiguate the multiple possible object matches. Consistent with research by Blades and Cooke (1994), this finding suggests an early ability to map on the basis of object matches but not relational correspondences.

These findings are consistent with prior research on analogy and similarity showing that children progress in the kinds of similarities they can appreciate within a given domain (e.g., Gentner \& Rattermann, 1991; Halford, 1992; Smith, 1984; Vosniadou, 1989). One key aspect of this development is a shift from appreciating direct similarities between objects to appreciating similarities between the relations that hold among objects. Gentner (1988; Gentner \& Rattermann, 1991) termed this progression the relational shift and suggested that it results from a deepening of domain knowledge. The claim that domain knowledge drives analogical development has received considerable support (Brown, 1989; Chen, 
Sanchez, \& Campbell, 1997; Gentner \& Rattermann, 1991; Gentner \& Toupin, 1986; Goswami, 1992; Inagaki, 1989, 1990; Inagaki \& Hatano, 1987; Kotovsky \& Gentner, 1996; Vosniadou, 1989; although see Halford, 1992, for a different account). The idea is that early in learning a given domain, children (and adults) generally attend to objects and their properties as the basis for similarity judgments. As children gain knowledge of relational structure, they become able to perceive similarities on the basis of common relational structures (e.g., Chen et al., 1997; Gentner \& Toupin, 1986).

As noted, Gentner and Rattermann (1991) argued that the relational shift is driven by gains in domain knowledge. How do these gains come about? Our interest here is in learning processes. We suggest that comparison experiences are a major contributor to the observed gains in relational insight. In the case of the model task, there is evidence for performance improvements due to previous model task experience (Marzolf \& DeLoache, 1994; Uttal et al., 1995). The question is exactly how this prior experience leads to improvement.

We propose that the process of making a comparison between two spaces can promote children's understanding of the common spatial relational schema; thereby facilitating performance on a later mapping task. The key to our proposal is modeling comparison as a structure-mapping process that-even if initially prompted by common perceptual features-acts to render common relational structure salient (Gentner, 1983; Gentner \& Markman, 1997). According to structure-mapping theory, the process of comparison first finds an initial set of local matches, including object matches, and then coalesces these commonalities into a maximal structurally consistent ${ }^{1}$ alignment (Falkenhainer, Forbus, \& Gentner, 1989; Forbus, Gentner, \& Law, 1995). An important constraint on the structural alignment process is systematicity: A predicate that is connected to other mappable predicates via higher-order relations is more likely to be mapped than an isolated predicate. Because of this systematicity preference, the structure-mapping process acts to promote common relational structure. By promoting a focus on relational commonalities, comparison can render a common relational system more salient and more separable from its objects and, thus, more available for further use (Gentner \& Medina, 1997; Gentner \& Namy, 1999; Gick \& Holyoak, 1983; Loewenstein \& Gentner, 1997; Loewenstein, Thompson, \& Gentner, 1999; Markman \& Gentner, 1993, 1997; Medin et al., 1993; Namy \& Gentner, in press). Indirect evidence for this claim comes from research showing that solving a pair of similar problems increases the likelihood that children will solve a further similar

\footnotetext{
${ }^{1}$ A structurally consistent alignment is one that maintains a one-to-one mapping (i.e., an element in one representation corresponds to, at most, one element in the other representation) and parallel connectivity (i.e., if predicates correspond across the two representations, then their arguments must correspond as well).
} 
problem (Brown \& Kane, 1988; Brown, Kane, \& Echols, 1986; Chen \& Klahr, 1999; Chen et al., 1997; Uttal et al., 1995). We have referred to the process of forming encodings based on comparison as analogical encoding (Ferguson, 1994; Kurtz, Miao, \& Gentner, in press; Loewenstein et al., 1999). Here we seek more direct evidence that analogical encoding facilitates learning and transfer.

The tack we are taking is different from that of prior research that focuses on $\mathrm{far}$ analogies-comparisons between examples that share relational content but little object similarity. Without denying the value of far analogies, we suggest that for novice learners, close analogies are also informative. Comparing highly similar examples that match on both objects and relations can facilitate abstracting a schema and later perceiving purely relational commonalities. For example, Kotovsky and Gentner (1996) found that 4-year-olds who carried out a same-dimension similarity choice task-for example, matching symmetry in size with symmetry in size-later performed successfully on an otherwise difficult cross-dimensional similarity choice task - for example, matching symmetry in size with symmetry in shading. These results are perhaps surprising: It may have been supposed that experience with highly similar examples would lead to the formation of a narrow understanding. Our suggestion is that highly similar examples have two advantages. First, they invite comparison because of their obvious surface similarities. Second, because the obvious object matches lead to correct correspondences, the child is likely to arrive at the correct alignment. The process of comparison then leads children to discover further relational similarities. Thus, our hypothesis is that comparing highly similar examples will help children encode relations more explicitly. This, in turn, will help them carry out further tasks-such as mapping tasks - involving these relations.

We can apply this line of reasoning to studies by Marzolf and DeLoache (1994). They found that children who performed a mapping task between two models of high object similarity later performed better on a mapping task with low-similarity models than children who had received low-similarity models in both sessions. These results allow for several interpretations. Experience with highly similar examples could have promoted task understanding, or it may have fostered the representational insight that one space can stand for another. We suggest that comparison could be the key. The high-similarity mapping task constituted an easily alignable comparison. By the account just given, the process of alignment led to greater attention to the common spatial configuration that characterized both rooms. Thus, when given a task with low object similarity (but the same spatial configuration), these children were able to use relational correspondences to augment the weak object matches.

Our research tests this account. We ask whether comparing two similar spaces facilitates children's ability to carry out a difficult mapping task over the same spatial relations. To do this, we first designed a challenging mapping task between two models. We then tested whether comparing highly similar model rooms pro- 
moted successful performance on this challenging mapping task. We also tested our prediction that comparison would lead to improved encoding of spatial relations by testing children's ability to retrieve the original toy.

We constructed a mapping task that tested children's ability to map on the basis of object and relational commonalities. Following Blades and Cooke (1994), both the hiding room and the finding room contained twin items - two identical stools - and unique items-a bed, a desk, and a rug. The mapping between the unique items tests children's ability to make object matches. The mapping between the twin items tests children's ability to use relational information to make matches. The objects in the finding room were of the same category and occupied the same relative positions as the corresponding objects in the finding room, but to increase task difficulty, they differed in shape and color. Finally, we made a high-similarity comparison room (the Hiding 2 room) that was nearly identical to the Hiding 1 room. Our goal was not to create a bridge but rather to create a high-similarity comparison pair. The prediction was that seeing two similar highly alignable rooms--Hiding 1 and Hiding 2would promote noticing the common system of spatial relations.

In Experiment 1, we showed children two similar hiding events, permitting a sequential comparison during the mapping task, which resulted in performance benefits relative to a control group. Experiment 2 showed a more striking effect of comparison: Simply comparing the two nearly identical hiding models initially helped children to succeed on the subsequent mapping task. Experiment 3 showed that the comparison benefit was not simply due to an opportunity to interact with two examples but was specific to comparing them.

\section{EXPERIMENT 1}

This experiment tested preschoolers' ability to solve a difficult mapping task with or without the opportunity to compare two highly similar rooms. Preschoolers are in the midst of substantial developmental changes in understanding space (Newcombe \& Huttenlocher, 2000). We focused on children aged 3 and $31 / 2$ years, a range over which rapid changes take place in children's spatial mapping abilities (Blades \& Cooke, 1994; DeLoache, 1987, 1989b). The children played a hiding and finding game, such as that used in the previous model studies (Blades \& Cooke, 1994; DeLoache, 1987, 1995). One half of the children were shown the hiding event in both the Hiding 1 and Hiding 2 rooms before searching for a toy in the finding room; the other half just saw it in the Hiding 1 room. We predicted that watching toys being hidden in both the Hiding 1 and Hiding 2 rooms would lead children to compare them, highlighting the spatial relational schema common to the rooms. A grasp of the common configuration should help children to make both the nonobvious object matches and the relational matches required to map between the Hiding 1 and finding rooms. Thus, children given an opportunity to compare the 
Hiding 1 and Hiding 2 rooms should perform better on the mapping task than a control group not given such experience. We had one further expectation, which was based on previous results (Blades \& Cooke, 1994) and consistent with the relational shift hypothesis. Children should perform better on the unique items, which only require object matches, than on the twin items, which require matches of both objects and spatial relations.

\section{Method}

Participants. Participants were 44 children from middle- and upper middleclass families in the northern metropolitan Chicago area. Four of the children did not complete the task and were excluded from the analyses. The 20 younger children ranged in age from 35 to 39 months $(M=37.4, S D=1.31)$. The 20 older children were aged 40 to 45 months $(M=42.2, S D=1.32)$. One half of the children were girls, and the other half were boys.

Design. In each of the two age groups, one half of the children were placed in the comparison condition and one half in the control condition. Each participant had to find toys at both unique and twin items. Thus, the design was a randomized factorial mixed design with three between-subjects factors-age (37 and 42 months), comparison (control or comparison), and gender-and one within-subject factor--object type (unique or twin). The dependent measures were the number of correct responses on the placement trial, on the four search trials in the finding room, and on the four retrieval trials in the Hiding 1 room. All children were allowed to search until they found the toys, but their responses were scored correct only if they searched first at the correct location.

Materials. There were three rooms, a Hiding 1 room, a Hiding 2 room, and a Finding room. All the rooms were 10 - $\mathrm{ft}$ high $\times 12.5$ - $\mathrm{ft}$ wide $\times 10$ - $\mathrm{ft}$ deep. The rooms had a full back wall, a full floor, and two diagonally cut side walls. There was no ceiling or front wall. Each was a different color. There were five items of furniture in each room: a bed, a desk, a rug, and two stools (the twin items). The corresponding objects in the Hiding 1 and Hiding 2 rooms were identical in shape and size and differed only in color. The corresponding objects in the Finding room came from the same basic-level categories but had different shapes, slightly different sizes, and were differently colored. A small, stuffed dog was placed in front of each room. Each dog had a flat, magnetic bone that the experimenter hid under items of furniture. Each piece of furniture had a magnet attached beneath it for this purpose (except for the rugs, which covered the flat dog bones adequately on their own), set such that the bone could not be seen when hidden. The Hiding 1, Hiding 2, and Finding rooms could be placed in a row on top of a table, arranged so that children could see into all of them at once (see Figure 1). 
Hidingl room

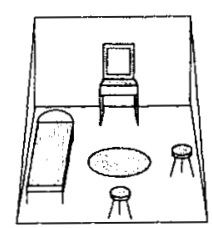

Hiding2 room

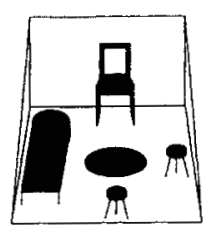

Finding room



FIGURE 1 The model rooms used in Experiment 1.

To ensure that the Hiding 1 and Hiding 2 rooms were highly similar to each other and equally different from the Finding room, 10 undergraduates at Northwestern University (given course credit for their participation) wrote pairwise ratings of similarity and chose which room was least like the other two. All 10 participants wrote that the finding room was most different from the other two rooms, and 9 of the 10 participants rated the Hiding 1 and Hiding 2 rooms to be more alike than any other pairing. The remaining participant gave all pairings the same rating. The mean rated similarity between the Hiding 1 and Hiding 2 rooms ( $M=6.3$ out of $7, S D=0.67$ ) was significantly greater than that between the Hiding 1 and Finding rooms $(M=4.6, S D=1.17), t(9)=4.630, p<.05$, and that between the Hiding 2 and finding rooms $(M=4.5, S D=1.27), t(9)=5.667, p<.05$. These results confirmed the close similarity between the Hiding 1 and Hiding 2 rooms and lower similarity between the Hiding 1 and Finding rooms. Importantly, the Hiding 2 room was no more similar to the Finding room than was the Hiding 1 room. Thus, it could not serve as a bridge between the Hiding 1 and Finding rooms.

Procedure. In both conditions, each child was first presented with a gray dog and its bone. The experimenter brought out the Hiding 1 room and a basket containing furniture and said, "This dog has a room, and in his room he puts his furniture." The experimenter continued, "He's got a bed, which he puts right here," while placing the bed in its position in the room. Each item of furniture was placed in the Hiding 1 room in this manner. In the comparison condition, the experimenter next brought out the Hiding 2 room, fully set up, and placed it to the right of the initial room. The experimenter said, "See, here is another dog, and he's got a room too, and his room is just the same." For all children, the experimenter then brought out the finding room, fully set up, saying, "See, here is another dog, and he's got a room too, and his room is just the same."

Placement trial. The children watched the experimenter perform a placement trial to ensure they were alerted to the relations between the rooms. The child was told, "These dogs like to put their bones in the same place. Sometimes this dog 
likes to put his bone right here," as the experimenter placed the bone on the rug in the Hiding 1 room. In the comparison condition, a bone was also placed in the Hiding 2 room as the experimenter said, "then this dog puts his bone right here." For all children, the experimenter said, "then this dog puts his bone right here" while placing a dog's bone on the rug in the finding room. The experimenter then removed all the bones from the rooms and said, "Another time this dog puts his bone right here," while placing a bone in the front left corner of the Hiding 1 room. In the comparison condition, the experimenter placed a bone in the same place in the Hiding 2 room, saying, "and this dog puts his bone right here." In both conditions, the experimenter asked the child about the finding room: "Can you put this dog's bone in the same place in his room?" If the child placed the toy in a very different place in the finding room, the experimenter asked the child if the bones were now in the same place but did not correct the child.

Search trials. Next, the child was invited to help hide bones for the dogs. The child and the experimenter put a bone under an object in the Hiding 1 room. If the child was in the comparison condition, the experimenter put a toy in the same place in the Hiding 2 room. Then, while the child closed his eyes, the experimenter hid a bone in the finding room. The experimenter said, when the child's eyes were open, "I hid this dog's bone in this room in the very same place that we just put the other one(s). Can you find this dog's bone?" The child searched the finding room until finding the bone, but the response was only counted as correct if the child found the toy on the first attempt. Four search trials were done, alternating between the unique items (i.e., the desk and bed) and the twin items (i.e., the two stools). Additionally, a criterion was set at being correct on at least three of the four trials as a measure of high individual performance (as in DeLoache, 1989b, and because it is, conservatively, reliably better than chance by a binomial).

Retrieval trials. On each trial, after the bone was found in the finding room, children retrieved the bone from the Hiding 1 room as a check on their understanding of the location of the original toy (DeLoache, 1987). The comparison group retrieved bones from the Hiding 1 and Hiding 2 rooms but was only scored for the first (Hiding 1) retrieval.

\section{Results}

Search trials. As predicted, children in the comparison condition $(M=0.69$ correct, $S D=.028)$ performed better than children in the control condition $(M=$ $0.49, S D=.032$ ). The overall low performance of children in the control condition confirmed that the mapping between the Hiding 1 and Finding rooms was difficult (see Table 1 and Figure 2). The data for search trials were analyzed in a 2 (age) $\times 2$ (comparison) $\times 2$ (gender) $\times 2$ (object type) mixed-measures analysis of variance 
TABLE

Experiment 1: Proportion Correct for the Search Trials

\begin{tabular}{llll}
\hline & \multicolumn{3}{c}{ Search Trials } \\
\cline { 2 - 4 } Group & Unique & Twin & Overall \\
\hline 36-month-olds & & & \\
$\quad$ Control & 0.45 & 0.35 & 0.40 \\
$\quad$ Comparison & 0.70 & 0.45 & 0.58 \\
42-month-olds & & & \\
$\quad$ Control & 0.70 & 0.45 & 0.58 \\
$\quad$ Comparison & 1.0 & 0.60 & $0.80^{* *}$ \\
All & & & \\
$\quad$ Control & 0.58 & 0.40 & 0.49 \\
$\quad$ Comparison & $0.85^{*}$ & 0.53 & $0.69^{*}$ \\
$M$ & 0.71 & 0.46 & 0.59 \\
\hline${ }^{*} p<.05 .{ }^{* *} p<.1$. & & &
\end{tabular}

(ANOVA). As mentioned previously, there was a main effect of comparison $F(1$, $32)=5.447, p<.05$. There was also a significant effect of age: Older children $(M=$ $.69, S D=.028)$ performed better than younger children $(M=.49, S D=.032), F(1$, $32)=5.447, p<.05$. This effect was moderated by a significant Age $\times$ Gender interaction, which showed that only girls showed an effect of age (36-month-olds: $M=$ $.40 ; 42$-month-olds: $M=.80$; as compared to $M=.58$ for both 36 - and 42 -month-old boys), $F(1,32)=5.447, p<.05$. Despite the overall difference in performance between boys and girls, both genders showed an advantage due to comparison $(0.73$ vs. 0.43 for boys and 0.65 vs. 0.55 for girls for comparison vs. control, respectively). We found no effects of feedback or practice across trials (consistent with Blades \& Cooke's, 1994, findings), and we did not find above-chance tendencies for children to make perseverative errors.

An analysis of individual performance showed a similar pattern as that of the group means. More comparison group children (.70) than control group children (.40) met a criterion of .75 correct. This finding held for both the younger children (comparison $=.50 ;$ control $=.30$ ) and older children (comparison $=.90$; control $=$ $.50)$.

As predicted, children performed better on the unique objects $(M=0.71$ correct, $S D=.019)$ than on the twin objects $(M=0.46, S D=.009), F(1,32)=18.182, p<.05$. All groups performed reliably above chance (all $t \mathrm{~s}>3.7$ ) except the younger control group children, who performed marginally above chance $(.20), t(9)=2.14, p=.06$. On the twin items, only the older comparison group performed reliably better than chance, $t(9)=4.00, p<.01$. Performance did not exceed chance for the younger control group, $t(9)=1.41, n s$, and was just marginally better than chance for the younger comparison group and older control group, $t(9)=2.14, p=.06$, for both. 
The effect of comparison was stronger for unique items than for twin items. On the unique items, comparison group children $(M=.85, S D=0.33)$ performed better than the control group children $(M=.58, S D=0.37), t(38)=2.476, p=.018$. On the twin items, the comparison group $(M=.53, S D=0.34)$ showed only a nonsignificant tendency to perform better than the control group $(M=.40, S D=$ $0.35), t(38)=1.268, n s$.

Twin items. We assessed the degree to which children in different conditions were able to make use of relational information to disambiguate the twins during search. If children base their search solely on object matches and if their object mapping were perfect, then when an object is hidden under one twin, they will choose randomly between the two twin objects. This predicts $50 \%$ correct performance. However, because children's object matching is not perfect, that figure must be adjusted. We can take performance on the unique items as an estimate of children's ability to match based on object characteristics. Thus, we derive the object-only estimate-that is, how children would perform on the twin items if they based their search solely on object matches - by multiplying the probability of a correct object match (i.e., the proportion correct on the unique items) by .50 (i.e., to represent random selection between the twin items). We can then test whether children's performance on the twin items was significantly higher than the object-only estimate.

In this study, children's performance on the twin items was well accounted for by the object-only estimate. The 37 -month-old control group children were $35 \%$ correct on the twin items, against an object-only estimate of $23 \%$; comparison group children were $45 \%$ correct, against an object-only estimate of $35 \%$. Among the 42-month-olds, the control group children were $45 \%$ correct against an objectonly estimate of $35 \%$, and the comparison group children were $60 \%$ correct against an object-only estimate of $50 \%$. The proportion correct did not differ significantly from the object-only estimate for any of the groups, all $t s<1.2$.

FIGURE 2 Proportion of correct first searches in Experiment 1 .

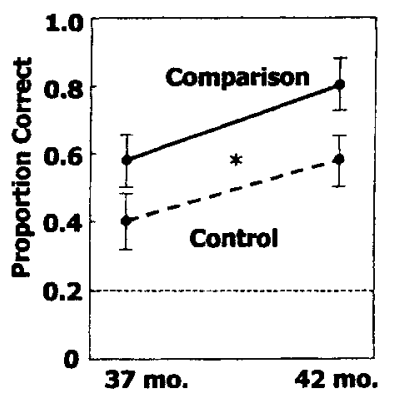


There was an unexpected asymmetry in performance on the twin items. Children were far more likely to choose the twin item at the front of the room correctly $(M=0.65)$ than the twin item at the side of the room $(M=0.28)$. We suspect this asymmetry was due to one of the twin items being placed at the front of the rooms (i.e., closer to the children), whereas the other was placed at the side-a disparity we remedied in the succeeding studies. Overall, children searched (correctly and incorrectly) at the front twin item on $43 \%$ of trials ( $20 \%$ more than at any other item) and at the side twin item on just $12 \%$ of trials.

Retrieval trials. The overall performance on the retrieval trials was good ( $M$ $=0.76$ correct, $S D=0.24)$, and it was higher on the search trials $(M=0.59, S D=$ 0.31 ). There was no effect of condition, suggesting that the advantage of the comparison group on the search trials was not primarily due to differences in the children's memory for the location of the original toy. However, there was an effect of object type: Although children performed above chance (.20) levels for both item types, all $t \mathrm{~s}>3.2$, children performed better on the unique items $(M=0.89, S D=$ $0.24)$ than on the twin items $(M=0.64, S D=0.34), F(1,32)=21.053, p<.05$. The high level of performance on the unique items by all groups of children suggests that they were on task. The poorer performance on the twin items suggests a particular disadvantage for encoding or recalling relational information. Consistent with this suggestion, all groups performed roughly in accord with the object-only estimate-all higher, but not significantly so.

As with the search trials, there was an asymmetry for the twin items on the retrieval trials. Children performed better on trials involving the front twin item $(M=$ $0.88)$ than those involving the side twin item $(M=0.40)$. Again, we suspect that the reason for the difference is that the front twin item was more easily accessible than the side twin item.

Search trial results for those trials on which children successfully retrieved the original toy show the same patterns (albeit at higher proportions) as the results from all the search trials. The comparison group children performed better $(M=$ $0.83)$ than the control group children $(M=0.59)$, older children $(M=0.80)$ performed better than younger children $(M=0.62)$, and children were more successful on the unique items $(M=0.77)$ than on the twin items $(M=0.64)$.

Placement trials. There was a significant effect of condition on the placement trials, with children in the comparison condition $(M=0.80$ correct) placing better than those in the control condition $(M=0.40), F(1,32)=7.529, p<.05$. There was an immediate effect of alignment on children's understanding of the correspondences between the hiding and finding rooms. 


\section{Discussion}

Our main prediction was confirmed: Comparison group children performed better than control group children. As predicted, comparing highly similar examples helped 3-year-olds to subsequently make a more challenging comparison. The superior performance of the comparison group is not easily explained by differences in children's memory for the location of the original toy, as children in both conditions performed at comparable levels on the retrieval trials.

Children performed far better on the unique items than on the twin items, consistent with the relational shift prediction that children achieve object matches before relational matches. The advantage of unique over twin items held for retrieval trials as well as search trials, suggesting that it results in part from an encoding advantage. That is, children did not encode the relational information necessary to distinguish the twin items. However, encoding failures are not sufficient to account for children's difficulties with the twin items. Children's search trial performance was better for the unique items than for the twin items even on just those trials in which children later retrieved the toy correctly in the original space. Thus, children's differential mapping performance on the twin versus unique items cannot be attributed merely to memory failure.

The results of Experiment 1 are encouraging for the claim that comparison facilitates encoding and mapping on the basis of relations. However, a question remains about the exact nature of the comparison benefit. Our hypothesis is that the advantage of the comparison group stems from comparing the highly similar Hiding 1 and Hiding 2 rooms and abstracting the common spatial relations, but the comparison group children could also have benefited from the repetition of the hiding event. The comparison group children saw toys being hidden in both the Hiding 1 and Hiding 2 rooms, whereas the control group children saw just one hiding event. The comparison group's superior performance may simply have stemmed from having two chances to view the hiding event and not from any insight into spatial schemas. Another concern was the extent of the comparison benefit. Despite similar trends for the unique and twin items, effects of comparison were reliable only for the unique items. If alignment invites relational focus, there should be benefits for the twin items.

\section{EXPERIMENT 2}

In Experiment 2, we sought to hone in on the pure effects of comparing two spaces. Instead of showing the hiding event twice, we simply showed comparison group children the nearly identical rooms-Hiding 1 and Hiding 2-before the hide-andfind task and encouraged them to point out how the two rooms were alike. A control group received initial experience with the Hiding 1 room prior to the mapping task. 
To equalize the time spent looking at the hiding room, the control group was encouraged to talk about the colors of the objects in the Hiding 1 room.

After their pretask experience, all children in the study played the same hideand-find game; the only difference between conditions was the pretask experience children were given. In addition to removing the double-event confound, this design gives the comparison group children a more focused opportunity to compare the two spaces. Because the comparison took place prior to the difficult mapping task, children could draw out a relational schema from their comparison experience before needing to apply it. Thus, it constitutes a purer test of the analogical encoding hypothesis.

If comparison, and not repetition, was the major factor responsible for the effects in Experiment 1, then when children are allowed a focused comparison in Experiment 2 , the comparison group should outperform the control group. In particular, if comparing highly similar examples promotes the encoding and transfer of relational structure, then comparison group children should exceed the control group children on the twin items.

As before, we also had expectations about performance across object types. The relational shift hypothesis - that the ability to make object matches precedes the ability to make relational matches-predicts that children should perform better on unique items than on twin items and that age differences should be most pronounced on the twin items, which require some understanding of spatial relations.

\section{Method}

Participants. Participants were 52 children from the same population as in Experiment 1. Four boys were unable to complete the task and were dropped from analysis, leaving 48 children in two age groups: 35 to 37 months $(M=36.2, S D=$ $0.41)$ and 40 to 44 months $(M=42.3, S D=1.15)$. One half of the participants were boys, and one half were girls. One half were placed in the comparison condition and one half in the control condition.

Design and materials. The design, scoring, and materials were as in Experiment 1, with one exception: The furniture was rearranged to equalize the salience of the two twin items. The front twin item was moved to the back of the room, between the bed and the desk, and the side twin remained in place. To corroborate equal salience, we ran a pilot study with 7 children ranging in age from 39 to 49 months ( $M=41$ months). The children closed their eyes while an experimenter hid a toy in the finding room, and then the children were asked to try to find it. Each child completed four trials - one for each unique and twin item used for the search trials in Experiment 1. The children searched for the toy equally often in the five hiding places in this new arrangement: the bed $(M=0.18)$; the desk $(M=0.22)$; the 
rug $(M=0.16)$; and, most important, the side stool $(M=0.19)$ and the back stool $(M$ $=0.21)$. Two children also looked under the $\operatorname{dog}(M=0.04$; a reasonable but unexpected guess for the location of a hidden bone!). It appears that no object is considerably more or less salient than any other in this new arrangement.

Procedure. The procedure was largely the same as in Experiment 1, with the differences noted previously. For the comparison group, the experimenter brought out both the Hiding 1 and Hiding 2 rooms and encouraged the children to compare them. First, the experimenter set up the objects in the Hiding 1 room as in Experiment 1 , then the Hiding 2 room was placed next to it with its objects already fully set up. The experimenter said, "Do you see how these are the same? Let's see how these are the same." The experimenter pointed to an object in the Hiding 2 room and asked the child to point to "the one in the very same place" in the Hiding 1 room. Children were given general encouragement but no specific feedback. After the experimenter and child went through all the objects in the room twice in this fashion, the Hiding 2 room was removed and the finding room was brought out.

For the control group children, the pretask involved only the Hiding 1 room. As with the comparison group, the experimenter brought out the Hiding 1 room and set up the objects. Then, the experimenter encouraged the child to talk about the colors of all the objects in the Hiding 1 room. This color-naming task was roughly the same duration as the comparison group's pointing task.

The experimenter then brought out the finding room. From this point on, both groups received exactly the same treatment: Children carried out two placement trials and four search and retrieval trials, much like children in the control condition of Experiment 1 . The two placement trials were at the front left and front right corners of the rooms. The four search and retrieval trials alternated between unique and twin items.

\section{Results}

Search trials. As predicted, the comparison experience helped children in the mapping task (see Table 2 and Figure 3 ). The data for search trials were analyzed in a 2 (age) $\times 2$ (comparison) $\times 2$ (gender) $\times 2$ (object type) ANOVA. There was a main effect of comparison: Children in the comparison condition $(M=0.65$ correct, $S D=0.23$ ) performed better than children in the control condition $(M=$ $0.47, S D=0.33), F(1,40)=5.372, p<.05$. There was a main effect of age, with older children $(M=0.64, S D=0.33)$ performing better than younger children $(M=$ $0.48, S D=0.24), F(1,40)=4.182, p<.05$. There was a marginal interaction of Age $\times$ Comparison: The 42-month-old comparison group performed substantially better $(M=0.79, S D=0.14)$ than the remaining three groups $(M=0.48, S D=0.30)$, $F(1,40)=3.141, p=.084$. There was also a marginal effect of gender: Overall, girls 
TABLE 2

Experiment 2: Proportion Correct for the Search Trials

\begin{tabular}{llll}
\hline & \multicolumn{3}{c}{ Search Trials } \\
\cline { 2 - 4 } Group & Unique & Twin & Overall \\
\hline 36-month-olds & & & \\
$\quad$ Control & .63 & .29 & .46 \\
$\quad$ Comparison & .79 & .21 & .50 \\
42-month-olds & & & \\
$\quad$ Control & .54 & .42 & .48 \\
$\quad$ Comparison & $.88^{*}$ & $.71^{*}$ & $.79 *$ \\
All & & & \\
$\quad$ Control & .58 & .35 & .47 \\
$\quad$ Comparison & $.83^{*}$ & .46 & $.65^{*}$ \\
$M \quad$ & .71 & .41 & .56 \\
\hline${ }^{*} p<.05$. & & &
\end{tabular}

$(M=0.63, S D=0.27)$ performed better than boys $(M=0.49, S D=0.32), F(1,40)=$ $3.141, p=.084$. However, there were no interactions with gender-both girls and boys showed benefits of comparison.

The pattern of search trial performance by individual children mirrored that of the group means. Few 36-month-olds met a 0.75 correct criterion (comparison $=0.17$, control $=0.25$ ). Among the 42 -month-olds, more than twice as many comparison group children (0.92) than control group children $(0.42)$ met criterion. The effects of alignment experience appear to have been stronger at 42 months than at 36 months.

There was a reliable effect of object type. Children performed better on the unique objects $(M=0.75$ correct, $S D=0.35)$ than on the twin objects $(M=.41, S D$ $=0.37), F(1,47)=45.166, p<.05$. Further validating the new furniture arrangement, children's performance was roughly equivalent on the two twin items (side stool: 0.44 correct; back stool: 0.38 correct). There was also an Object Type $\times$ Age interaction, $F(1,47)=11.795, p<.05$. Although children performed well on the unique items regardless of age ( 36 months: $M=0.75$ correct; 42 months: $M=0.74$ correct), only the older children showed any measure of success for the twin items (36 months: $M=0.23 ; 42$ months: $M=0.57$ ). Comparisons with chance performance bore out this pattern of results. Although all groups performed above chance $(0.20)$ on the unique items, all $t s>2.6$, only the older children exceeded chance on the twin items-reliably so for the older comparison group, $t(11)=6.84$, $p<.001$, and marginally so for the older control group, $t(11)=2.09, p=.06$.

A key question was whether comparison promoted relational understanding sufficiently to improve performance on twin items (see Figure 4). This advantage was found for the older children. The 42-month-olds in the comparison group ( $M=$ $0.71, S D=0.26$ ) performed better on the twin items than those in the control group 
$(M=0.42, S D=0.36), t(22)=2.288, p<.05$. No such advantage was found for the younger children. Additionally, only the 42-month-old comparison group ( $M=$ $0.71, S D=0.26$ ) was correct on the twin items significantly more than predicted by its object-only estimate $(0.44), t(11)=3.644, p<.05$. Making a concrete comparison apparently led at least the older children to greater insight of relational correspondences.

Comparison experience also appears to have aided children in making the object matches. Although all groups performed above chance on the unique objects, the comparison group $(M=0.83, S D=0.28)$ performed better than the control group $(M=0.58, S D=0.38), t(46)=2.584, p=.013$. It appears that comparing highly similar examples can help children make low-similarity object matches. We suspect this happens for two reasons: First, the alignment process acts to preserve object commonalities as well as relational commonalities, and, second, having a firmer grasp of relational correspondences helps children to buttress the object correspondences.

Retrieval trials. Overall, children showed good memory for where the bone was hidden in the original Hiding 1 room $(M=0.83$ correct, $S D=0.21)$, and all groups performed above chance $(0.20)$ levels on both the unique and twin items, all

FIGURE 3 Proportion of correct first searches in Experiment 2.

FIGURE 4 Proportion of correct first searches for the twin items in Experiment 2
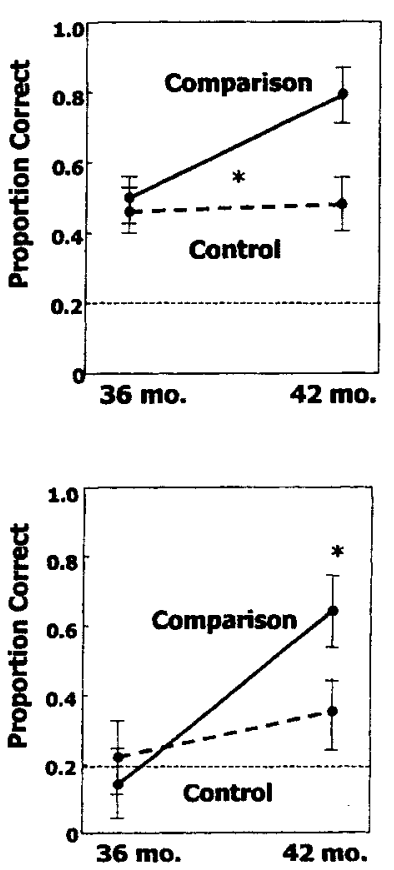
$t \mathrm{~s}>3$.9. There was a marginal effect of age, with the older children performing at $89 \%$ correct $(S D=0.21)$, but even the younger children performed reasonably well at $78 \%(S D=0.21), F(1,40)=2.976, p=.092$. There was also a marginal effect of object type, as children performed better on the unique items $(M=0.89, S D=0.24)$ than on the twin items $(M=0.78, S D=0.32), F(1,40)=3.571, p=.066$. The object type effect suggests that the twin items present an added difficulty relative to the unique items - specifically, the need to encode spatial relations. The younger control group children showed some difficulty encoding spatial relations. They did not perform significantly better than the object-only estimate (all other groups performed better than the object-only estimates), all $t \mathrm{~s}>3.5$.

Placement trials. Performance on the placement trials was fairly low overall, at $43 \%$ correct $(S D=0.50)$. The 42 -month-olds in the comparison condition did best, performing at $59 \%$ correct, but there were no significant differences of age or condition, and there was no interaction of these factors. It is possible that the low performance was due to the location of the added placement trial, which was not clearly situated next to any one object.

\section{Discussion}

These results support the claim that comparing highly similar examples can promote highlighting the common relational structure, thereby facilitating a difficult relational mapping. Of most interest, the 42-month-old comparison group was successful on the twin items, showing an understanding of relational correspondences. Making a comparison helped children (at least the older children) to grasp a spatial relational schema.

The success of the comparison group children is of particular interest because all the children were tested on the same task. The only difference between conditions was the pretask game children experienced. Viewing one hiding space separately and talking about the furniture did not improve performance on the search task (indeed, the performance of the control group [47\% correct] in Experiment 2 was comparable to that of the control group [0.49] in Experiment 1). However, encouraging children to compare two nearly identical spaces provided substantial help.

The results of Experiment 2 also shed light on the possible interpretations of Experiment 1 . The findings show clear benefits from comparing two spaces. Although they do not rule out the possibility that the comparison benefit in Experiment 1 simply resulted from seeing a repeated hiding event, they show that such repetition is not necessary-simply comparing the two spaces before any hiding events are shown is sufficient to achieve the comparison advantage. Another salient point is that only in Experiment 2 did we find a comparison advantage on the twin items - a key measure of relational insight. The pure comparison manipula- 
tion in Experiment 2 led to greater gains than the double-event technique of Experiment 1 (although only for the older group). This gain may result from the greater opportunity to focus fully on the comparison in Experiment 2.

Overall, the results are encouraging. In both studies, comparison group children performed better than control group children, and yet there remains a key question. The comparison group saw two models prior to the finding task, perhaps conferring an extra advantage over and above comparing the rooms. That is, perhaps they simply learned the layout better as a result of interacting with two rooms instead of just one. To test this, we conducted a further study in which all children saw the Hiding 1 and Hiding 2 rooms, but only those in the comparison group were encouraged to compare them.

\section{EXPERIMENT 3}

Experiment 2 found a beneficial effect of comparison on appreciating relational similarity. The goal of this study was to replicate this effect as well as to rule out the possibility that mere exposure to two rooms, rather than the opportunity for comparison, can account for the comparison group's success. The comparison children saw the two hiding rooms simultaneously, as in the previous study. The control children saw the two hiding rooms sequentially and were encouraged to discuss the functions of all the objects in each room, one room at a time. Thus, the control group children saw exactly the same two rooms as the comparison group children and performed a relatively interesting task with each. The major difference was that only the comparison group saw the rooms simultaneously and were encouraged to compare them. One final change from the prior study is that the twin items were changed from stools to tables for generality.

\section{Method}

Participants. Participants were 50 children from the same population as in the previous experiments. Two 36-month-olds, 1 boy and 1 girl, were unable to complete the task and were dropped from analysis. The remaining younger children ranged in age from 35 to 37 months $(M=36.1, S D=0.78)$. The older children were aged 41 to 43 months $(M=42.3, S D=0.87)$. There were equal numbers of boys and girls, and one half were placed in the comparison condition and one half in the control condition.

Design and materials. The design, scoring, and materials were identical to those of Experiment 2, except that the stools were altered to look like small tables. In the Hiding 1 and Hiding 2 rooms, the tables were round and covered with table- 
TABLE 3

Experiment 3: Proportion Correct for the Search Trials

\begin{tabular}{llll}
\hline & \multicolumn{3}{c}{ Search Trials } \\
\cline { 2 - 4 } Group & Unique & Twin & Overall \\
\hline 36-month-olds & & & \\
$\quad$ Control & .58 & .29 & .44 \\
$\quad$ Comparison & .79 & .54 & $.67^{* *}$ \\
42-month-olds & & & .69 \\
$\quad$ Control & .79 & .58 & $.88^{* *}$ \\
$\quad$ Comparison & .92 & $.83^{* *}$ & .56 \\
All & & & $.77^{*}$ \\
$\quad$ Control & .69 & .44 & .67 \\
$\quad$ Comparison & $.85^{* *}$ & $.69^{*}$ & \\
$M \quad$ & .77 & .56 & \\
\hline$* p<.05 . * p<.1$. & & &
\end{tabular}

cloths that draped over the edges. In the finding rooms, the tables were short and boxy. This change provided a check on whether there was something particularly difficult about the stools we used in the previous experiments.

Procedure. The procedure for comparison group children was as in Experiment 2 . With the two hiding rooms side by side, the experimenter pointed to objects in the Hiding 1 room, and the child pointed to the corresponding objects in the Hiding 2 room. In contrast, there were changes made in the control condition procedure. The experimenter first set up the Hiding 1 room and asked the child what the dog did with each object in the room. The children could either answer verbally or demonstrate. After going through all the objects in the Hiding 1 room, the experimenter removed that room and brought out the Hiding 2 room, fully set up. The function game was played with this room in the same fashion. For both groups, after this pretask, the experimenter removed the Hiding 2 room and set up the Hiding 1 and finding rooms. The experimenter and child proceeded through placement, search, and retrieval trials as in Experiment 2.

\section{Results}

Search trials. As in the previous studies, there were clear benefits of comparison (see Table 3 and Figure 5). The search trial data were analyzed in a 2 (age) $\times$ 2 (comparison) $\times 2$ (gender) $\times 2$ (object type) ANOVA. As in the previous experiments, children in the comparison condition $(M=0.77$ correct, $S D=0.28)$ performed better than children in the control condition $(M=0.56, S D=0.31), F(1,40)$ 
$=6.250, p<.05$. There was a main effect of age, as the older children $(M=0.78, S D$ $=0.27)$ performed better than the younger children $(M=0.55, S D=0.31), F(1,40)$ $=7.563, p<.05$. There were no effects of, or interactions with, gender.

There was also an effect of comparison for the number of individual children meeting the $75 \%$ correct criterion, and this effect held for both age groups. Among 36 -month-olds, $67 \%$ of the comparison group met criterion, as compared to $25 \%$ in the control group. Among 42-month-olds, $92 \%$ of the comparison group met criterion, as compared to $67 \%$ in the control group.

As expected, performance was better on the unique objects $(M=0.77$ correct, $S D=0.34)$ than on the twin objects $(M=0.56, S D=0.38), F(1,40)=15.152, p<$ .05 . All groups performed above chance on the unique items, all $t \mathrm{~s}>3.7$. Still, there was a marginally significant tendency for children in the comparison condition $(M=0.85, S D=0.28)$ to outperform children in the control condition $(M=$ $0.69, S D=0.38$ ) on the unique objects, $t(46)=1.726, p=.092$.

On the twin items, two important differences in performance were reliable (see Figure 6). First, the comparison group $(M=0.69, S D=0.38)$ performed better than the control group $(M=0.44, S D=0.34), t(46)=2.847, p=.021$, consistent with the claim that a concrete comparison can foster relational insight. Second, older children $(M=0.71, S D=0.33)$ performed better than younger children $(M=0.42, S D$ $=0.38), t(46)=2.847, p=.007$, consistent with the relational shift hypothesis.

FIGURE 5 Proportion of correct first searches in Experiment 3 .

FIGURE 6 Proportion of correct first searches for the twin items in Experiment 3.

As in Experiment 2, the older group appeared to show stronger gains in relational insight than the younger group. Among the 42-month-olds (but not the 36month-olds), the comparison group $(M=0.83, S D=0.25)$ showed a marginally significant advantage on the twin items over the control group $(M=0.58, S D=$ $0.36), t(22)=1.990, p=.059$. The 42 -month-old comparison group was the only group that exceeded its object-only estimate $(0.46), t(11)=5.277, p<.05$. Thus, although comparison experience benefited both age groups, it led to clear relational gains for the older group.

Retrieval trials. Children performed well on the retrieval trials $(M=0.80$ correct, $S D=0.18$ ). All groups performed above chance $(0.20)$ for both unique and twin items, all $t \mathrm{~s}>2.8$. Nonetheless, children in the comparison group $(M=0.84, S D=$ $0.18)$ performed marginally better than children in the control group $(M=0.75, S D=$ $0.18), F(1,40)=3.24, p=.079$, suggesting an effect of comparison on children's initial encoding of the spaces. As in the previous studies, children performed better on unique items $(M=0.94, S D=0.17)$ than on twin items $(M=0.66, S D=0.36), F(1,40)$ $=20.138, p<.001$. There was a trend toward better performance on the twin items in the comparison group $(M=0.73, S D=0.29)$ than in the control group $(M=0.58, S D=$ 0.41 ), although the interaction did not reach significance.

Placement trials. The 42-month-old children $(M=0.79, S D=0.41)$ performed better than the 36-month-olds $(M=0.44, S D=0.50), F(1,40)=8.975, p<$ .05 . There was no effect of comparison.

\section{Discussion}

This study was a strong test of the hypothesis that making a comparison improves children's understanding of relational correspondences. Although comparison and control group children saw the same two initial rooms, only comparison group children were encouraged to compare them. This concerted comparison experience led to high levels of performance, especially among the 42-month-olds, who showed a clear advantage on twin items over the control group. This is evidence that comparing two scenes can enable children to succeed in a challenging relational mapping.

\section{FURTHER ANALYSIS OF THE TWIN ITEMS}

In previous studies using twin items (Blades \& Cooke, 1994; see also Marzolf \& DeLoache, 1997), children's performance was tested against an estimate of chance (0.50) based on the assumption that for each twin test trial, children who relied on object similarity alone would choose randomly between just the twin items. These studies maintained high object similarity across rooms, so that errors on twin trials 
were almost always to the wrong twin item. In contrast, in our studies, the object similarity across rooms was low so that on twin trials, children often searched at other (nontwin) objects. Across the three studies, children showed only modest proportions of errors to the wrong twin item on the search trials ( 0.41 of all errors) and retrieval trials $(0.51)$. These proportions of errors to the wrong twin item are consistent with those predicted by the object-only estimates $(0.37$ and 0.45 for the search and retrieval trials, respectively). ${ }^{2}$

We carried out a further test of whether children could distinguish between the twin items. For each child, we took the number of correct twin searches and divided it by the total number of twin searches for initial hidings under a twin. The average of these scores measures whether, given that children realized that one twin or the other is involved, they could tell which twin is correct (the few children who made no twin searches were dropped from this analysis). We tested this $p$ (correct twin and twin search) against the 0.50 chance measure.

This analysis revealed that in all three studies, the older children in the comparison group selected the correct twin at above-chance levels. Furthermore, in Experiment 3 , the younger comparison group (as well as the older control group) were able to select the correct twin (see Table 4). This analysis provides further support for the conclusion that comparison helped 42-month-olds to map on the basis of spatial relations.

\section{GENERAL DISCUSSION}

Our hypothesis is that the process of comparison-of structural alignment and mapping - can promote children's understanding of relational structure. Specifically, we hypothesized that comparison of analogous spatial configurations would garner attention to the common spatial relations and thereby facilitate relational encoding and performance in a subsequent spatial mapping task. The prediction was confirmed. In three studies, children who were led to compare two highly similar model rooms went on to perform better on a spatial mapping task than children not given such experience.

Comparison experience was provided in two different ways in these experiments. In Experiment 1, children watched two sequential hiding events before searching for a toy in the finding room. In Experiments 2 and 3, children compared two similar hiding rooms prior to seeing the hiding event and carrying out the

\footnotetext{
${ }^{2}$ In support of this explanation, in a recent pilot study in which 36-month-olds performed a mapping task between the high-similarity Hiding 1 and Hiding 2 rooms, we found that children were nearly perfect on the unique items, and nearly all (0.91) of children's errors on the twin items were to choose the wrong twin item.
} 
TABLE 4

Experiments 1 to 3: Proportion Correct Among the Twin Items on Twin Hiding Trials

\begin{tabular}{lccc}
\hline & \multicolumn{3}{c}{ Search Trials } \\
\cline { 2 - 4 } Group & Experiment 1 & Experiment 2 & Experiment 3 \\
\hline 36-month-olds & & & \\
Control & .44 & .56 & .50 \\
Comparison & .63 & .44 & $.94^{\mathrm{a}}$ \\
42-month-olds & & & \\
Control & .71 & .72 & $.80^{\mathrm{a}}$ \\
Comparison & $.72^{\mathrm{a}}$ & $.83^{\mathrm{a}}$ & $.96^{\mathrm{a}}$ \\
\hline
\end{tabular}

a95\% confidence interval does not include the chance value or .50 (proportion of correct twin searches among all twin searches).

search task. Both sequential comparison and pretask comparison resulted in performance benefits. Interestingly, pretask comparison appeared to lead to greater benefits than sequential comparison, as only the former was found to support children's relational mapping ability. This is consistent with other evidence suggesting that a concerted comparison process leads to better schema abstraction than does incidental comparison (e.g., Kurtz et al., in press; Markman \& Gentner, 1993; Reeves \& Weisberg, 1994).

Examining children's performance on the retrieval trials suggests that comparison benefits children's encoding of relations. Overall, children performed better on the unique than the twin items in their retrieval trials, consistent with our hypothesis that children's initial encodings of spatial relations were weak. However, we found that alignment experience-in particular, the opportunity for simultaneous comparison provided in Experiments 2 and 3-led to benefits on the twin item retrieval trials. This is consistent with our claim that analogical encoding - even the aligning of highly similar rooms - can improve children's relational encoding.

In all these studies, there were reliable benefits of comparison across both age groups. However, the effects of comparison were stronger for the older group than for the younger group. There was a reliable benefit of comparison for the older children in Experiment 2 and a marginal benefit in Experiment 3. In contrast, the younger children only showed a marginal benefit of comparison in Experiment 3 . Furthermore, only the older group showed benefits of comparison on the twin items - the key test of relational insight. It is possible that making comparisons was of greater benefit to the older children because for comparisons to yield insight, children must possess at least partial knowledge of the examples being compared. An additional possible factor is that the comparison experiences provided in these studies were brief. It is possible that more intensive comparison experience may enable younger children to gain insight into relations. 


\section{Alignment and Relational Learning}

Our results suggest that many transfer findings can be viewed as effects of making comparisons. For example, comparison-driven abstraction processes can help explain why performing an easy mapping task can facilitate performing a more challenging mapping task (Kotovsky \& Gentner, 1996; Marzolf \& DeLoache, 1994; Uttal et al., 1995). Furthermore, the assumption of analogical encoding processes can explain the finding that transfer is often markedly better if learners first solve two or more analogous problems, rather than just one-a result found for both children (Brown \& Kane, 1988; Chen et al., 1997; Gholson, Dattel, Morgan, \& Eymard, 1989; Gholson, Eymard, Morgan, \& Kamhi, 1987) and adults (Gick \& Holyoak, 1983; Loewenstein et al., 1999; Ross \& Kennedy, 1990; Thompson, Gentner, \& Loewenstein, 2000). For instance, Gholson and his colleagues (Gholson et al., 1989; Gholson et al., 1987) found that elementary school children could better solve a complex scheduling problem (e.g., the farmer's dilemma problem: ferrying a fox, a goose, and some corn across a river without leaving the fox with the goose or the goose with the corn) if they had received two prior analogs than if they had received only one. The benefits of comparison experience can be quite durable. Chen and Klahr (1999) taught 10-year-olds about experimental design by leading them to carry out an intensive sequence of parallel experiments (i.e., providing opportunities to compare highly similar examples) in the context of studying mechanics. Seven months later, the children showed positive transfer to novel domains.

Gentner and Namy (1999) found a comparison advantage on a word extension task. They found that when 4-year-olds were given a new name for a pictured object (e.g., an apple) and asked to extend the word to new objects, children extended the word to objects with common perceptual properties, such as shape (e.g., a balloon), rather than objects of with deeper conceptual commonalities (e.g., a banana). However, if children instead were shown two perceptually similar pictures with the same label (e.g., an apple and a pear) and were prompted to make a comparison, they shifted to extending on the basis of relational properties, such as both being edible (i.e., the banana received the same label as the apple). Comparing highly similar examples can enable children to note deep relational commonalities.

\section{Comparison and the Relational Shift}

The pattern of performance in our task is consistent in two respects with the relational shift hypothesis that in any given arena, facility with object commonalities precedes facility with relational commonalities (Gentner, 1988; Gentner \& Rattermann, 1991). First, in the studies reported here, children performed better on the unique items than on the twin items. Second, older children performed better 
than younger children on the twin items (which require relational encoding). The relational shift occurs at different ages in different domains (Gentner \& Rattermann, 1991). It appears in tasks involving similarity, analogy, and metaphor (e.g., Brown, 1989; Gentner, 1988; Gentner \& Rattermann, 1991; Gentner \& Toupin, 1986; Halford, 1987, 1992; Holyoak, Junn, \& Billman, 1984; Rattermann \& Gentner, 1998a, 2001). Interestingly, recent research suggests that the relational shift may also apply to the language children use to describe models. Plumert and her colleagues (Plumert, Ewert, \& Spear, 1995; Plumert \& Nichols-Whitehead, 1996) studied children's speech about object locations by hiding objects in model rooms that contained identical pairs of objects. They found that 3 -year-olds often mentioned the location of a toy but typically failed to mention the secondary landmarks needed to disambiguate which of two identical referents the child had intended. In contrast, 4year-olds tended to mention the relation to a secondary landmark.

Our studies add to evidence that the shift from an object focus to a relational focus is a general pattern resulting from greater expertise (Blades \& Cooke, 1994; Brown, 1989; Chen et al., 1997; Gentner \& Rattermann, 1991; Rattermann \& Gentner, 1998b). Prior evidence for this claim has come from correlations between children's knowledge in the domain and children's analogical abilities (Goswami, 1992; Goswami \& Brown, 1989; Rattermann \& Gentner, 1998b). These studies have the drawback that it is difficult to separate gains in experience from maturational gains. Furthermore, they do not tell us what kind of experiences lead to the relational shift. In this research, we provided children with comparison learning experiences that were hypothesized to lead to gains in relational knowledge. These experiences led directly to advantages in the ability to carry out relational similarity mappings. In other words, a relational shift occurred as a direct result of learning. In addition to buttressing the knowledge-gain explanation of the relational shift, these results bear on how relational knowledge is acquired. In our studies, we did not teach new facts; we simply encouraged comparison processing. Thus, we suggest that making comparisons is one experiential route by which children can grasp relational knowledge.

\section{Relational Schemas in Learning and Development}

Comparison is not the only route by which children develop an understanding of domain relations. Explanation and instruction can also promote relational learning (Bielaczyc, Pirolli, \& Brown, 1995; Callanan, 1990; Coleman, Brown, \& Rivkin, 1997; Crowley \& Siegler, 1999; Mayer, 1992; Palincsar, \& Brown, 1984). For example, Callanan (1990) found that parents helped 2- through 4-year-olds notice generalizable properties of examples when teaching their children new categories. Gentner and Toupin (1986) found in a story-mapping task that hearing a moral or causal schema that provided a higher order structure improved 9-year-olds' ability to retell the story with a different set of characters. Children can also profitably gen- 
erate their own explanations: Crowley and Siegler (1999) found that kindergarten through second grade students, who simply observed a strategy being used, were less successful at using it than were students who were also required to explain the strategy to themselves. Finally, children can capitalize on explanatory structure provided directly within learning materials.

One reason explanation may facilitate learning relational knowledge is that it may provide names for specific relations. Hearing relational language leads children to high levels of performance on spatial mapping tasks (Gentner \& Loewenstein, in press; Gentner \& Rattermann, 1991; Loewenstein \& Gentner, 1998; Rattermann \& Gentner, 1998a, 2001). For instance, we found that hearing locations described with spatial prepositions enabled 44-month-old children to succeed on a spatial mapping task in which control children performed at chance levels (Loewenstein \& Gentner, 1998).

Relations can be highlighted visually as well as verbally. For instance, lines on a map or graph can help make particular patterns or trends explicit (Zacks \& Tversky, 1999). Uttal and his colleagues (Tan \& Uttal, 1999; Uttal, 2000; Uttal, Gregg, Tan, Chamberlin, \& Sines, 2001) have extended the idea of structural highlighting to children's map understanding. They showed 5-year-olds where a toy was hidden on a map, and then asked the children to find the toy in a large-scale space. Children benefited from seeing a map with lines connecting the hiding locations. A follow-up study showed that children only benefited when the lines formed a coherent shape (e.g., a dog), rather than a meaningless figure (e.g., scrambled parts of a dog figure). Therefore, presenting a clear visual structure facilitated children's spatial mapping ability. This is consistent with Gentner's (1983; Clement \& Gentner, 1991) systematicity principle, according to which the comparison process is facilitated by common higher order structure.

\section{CONCLUSIONS}

The appreciation of relational similarities is a major achievement of development. The studies presented here suggest that the comparison process is one route to furthering children's appreciation of relational similarity. An intriguing aspect of the studies is that the comparisons that helped children were among highly similar examples. Studies of analogical learning have often focused on far analogies involving examples from widely different contexts. Such far comparisons can lead to dramatic results when people create abstract schemas that incorporate the relational commonalities. However, our research shows that the other end of the similarity continuum is also worth exploring. Children can gain insight from comparing highly similar pairs-the very kinds of comparisons they would naturally notice in the course of their own experience. The small step of a close comparison enabled the large step of a more distant analogy. 


\section{ACKNOWLEDGMENTS}

This article was partially prepared while Dedre Gentner was a Fellow at the Center for Advanced Study in the Behavioral Sciences.

This research was supported by National Science Foundation Grant SBR9720313 and National Science Foundation-Learning and Intelligent Systems Grant SBR-9511757 to Dedre Gentner. We are grateful for the financial support provided by William T. Grant Foundation Award 95167795. We thank Patricia Bauer, Zhe Chen, and Nora Newcombe for their thoughtful comments on the article. We also thank David Uttal, Judy DeLoache, Mark Blades, Phillip Wolff, and the Analogy and Similarity group at Northwestern University for helpful discussions of the issues presented in this article.

\section{REFERENCES}

Bence, V. M., \& Presson, C. C. (1997, April). The spatial basis of young children's use of scale models. Paper presented at the Biennial Meeting of the Society for Research in Child Development, Washington, $\mathrm{DC}$.

Bielaczyc, K., Pirolli, P. L., \& Brown, A. L. (1995). Training in self-explanation and self-regulation strategies: Investigating the effects of knowledge acquisition on problem solving. Cognition and Instruction, 13, 221-252.

Blades, M., \& Cooke, Z. (1994). Young children's ability to understand a model as a spatial representation. Journal of Genetic Psychology, 155, 201-218.

Blades, M., \& Spencer, C. (1994). The development of children's ability to use spatial representations. In H. W. Reese (Ed.), Advances in child development and behavior (pp. 157-199). San Diego, CA: Academic.

Bluestein, M., \& Acredolo, L. (1979). Developmental changes in map reading skill. Child Development, 50, 691-697.

Brown, A. L. (1989). Analogical learning and transfer: What develops? In S. Vosniadou \& A. Ortony (Eds.), Similarity and analogical reasoning (pp. 369-412). New York: Cambridge University Press.

Brown, A. L., \& Kane, M. J. (1988). Preschool children can learn to transfer: Learning to learn and learning from example. Cognitive Psychology, 20, 493-523.

Brown, A. L., Kane, M. J., \& Echols, C. H. (1986). Young children's mental models determine analogical transfer across problems with a common goal structure. Cognitive Development, I, 103-121.

Callanan, M. A. (1990). Parents' descriptions of objects: Potential data for children's inferences about category principles. Cognitive Development, 5, 101-122.

Chen, Z., \& Klahr, D. (1999). All other things being equal: Acquisition and transfer of the control of variables strategy. Child Development, 70, 1098-1120.

Chen, Z., Sanchez, R. P., \& Campbell, T. (1997). From beyond to within their grasp: The rudiments of analogical problem solving in 10- and 13-month-olds. Developmental Psychology, 33, 790-801.

Clement, C. A., \& Gentner, D. (1991). Systematicity as a selection constraint in analogical mapping. Cognitive Science, 15, 89-132.

Coleman, E. B., Brown, A. L., \& Rivkin, I. D. (1997). The effect of instructional explanations on learning from scientific texts. Journal of the Learning Sciences, 6, 347-365.

Crowley, K., \& Siegler, R. S. (1999). Explanation and generalization in young children's strategy learning. Child Development, 70, 304-316. 
DeLoache, J. S. (1987). Rapid change in the symbolic functioning of very young children. Science, 238 , $1556-1557$.

DeLoache, J. S. (1989a). The development of representation in young children. In H. W. Reese (Ed.), Advances in child development and behavior (Vol. 22, pp. 1-39). New York: Academic.

DeLoache, J. S. (1989b). Young children's understanding of the correspondence between a scale model and a larger space. Cognitive Development, 4, 121-139.

DeLoache, J. S. (1990). Young children's understanding of models. In R. Fivush \& J. A. Hudson (Eds.), Knowing and remembering in young children (pp. 94-126). Cambridge, England: Cambridge University Press.

DeLoache, J. S. (1995). Early symbol understanding and use. In D. Medin (Ed.), The psychology of learning and motivation (Vol. 32, pp. 65-114). New York: Academic.

DeLoache, J. S., Kolstad, V., \& Anderson, K. N. (1991). Physical similarity and young children's understanding of scale models. Child Development, 62, 111-126.

DeLoache, J. S., Miller, K. F., \& Rosengren, K. S. (1997). The credible shrinking room: Very young children's performance with symbolic and nonsymbolic relations. Psychological Science, 8, 308-313.

Dow, G. A., \& Pick, H. L. (1992). Young children's use of models and photographs as spatial representations. Cognitive Development, 7, 351-363.

Falkenhainer, B., Forbus, K. D., \& Gentner, D. (1989). The structure-mapping engine: Algorithm and examples. Artificial Intelligence, 41, 1-63.

Ferguson, R. W. (1994). MAGI: A model of analogy-based encoding using symmetry and regularity. Proceedings of the I6th Annual Conference of the Cognitive Science Society. Hillsdale, NJ: Lawrence Erlbaum Associates, Inc.

Forbus, K. D., Gentner, D., \& Law, K. (1995). MAC/FAC: A model of similarity-based retrieval. Cognitive Science, 19, 141-205.

Gentner, D. (1983). Structure-mapping: A theoretical framework for analogy. Cognitive Science, 7. $155-170$

Gentner, D. (1988). Metaphor as structure-mapping: The relational shift. Child Development, 59, 4759.

Gentner, D. (1989). The mechanisms of analogical learning. In S. Vosniadou \& A. Ortony (Eds)., Similarity and analogical reasoning (pp. 199-241). London: Cambridge University Press.

Gentner, D., \& Loewenstein, J. (in press). Relational thinking and relational language. In J. Bymes \& E. Amsel (Eds.), Language, literacy, and cognitive development Mahwah, $\mathrm{NJ}$ : Lawrence Erlbaum Associates, Inc.

Gentner, D., \& Markman, A. B. (1997). Structure mapping in analogy and similarity. American Psychologist, 52, 45-56.

Gentner, D., \& Medina, J. (1997). Comparison and the development of cognition and language. Cognitive Studies: Bulletin of the Japanese Cognitive Science Society, 4, 112-149.

Gentner, D., \& Namy, L. (1999). Comparison in the development of categories. Cognitive Development. 14, 487-513.

Gentner, D., \& Rattermann, M. J. (1991). Language and the career of similarity. In S. A. Gelman \& J. P. Byrnes (Eds.), Perspective on thought and language: Interrelations in development (pp. 225-277). New York: Cambridge University Press.

Gentner, D., \& Toupin, C. (1986). Systematicity and surface similarity in the development of analogy Cognitive Science, 10, 277-300.

Gholson, B., Dattel, A. R., Morgan, D., \& Eymard, L. A. (1989). Problem solving, recall, and mapping relations in isomorphic transfer and nonisomorphic transfer among preschoolers and elementary school children. Child Development, 60, 1172-1187.

Gholson, B., Eymard, L. A., Morgan, D., \& Kamhi, A. G. (1987). Problem solving, recall and isomorphic transfer among third-grade and sixth-grade children. Journal of Experimental Child Psychol$o g y, 43,227-243$ 
Gick, M. L., \& Holyoak, K. J. (1983). Schema induction and analogical transfer. Cognitive Psychology, $15,1-38$.

Goswami, U. (1992). Analogical reasoning in children. Hillsdale, NJ: Lawrence Erlbaum Associates, Inc.

Goswami, U., \& Brown, A. L. (1989). Melting chocolate and melting snowmen: Analogical reasoning and causal relations. Cognition, 35, 69-95.

Halford, G. S. (1987). A structure-mapping approach to cognitive development. The neo-Piagetian theories of cognitive development: Toward an interpretation [Special issue]. International Journal of Psychology, 22, 609-642.

Halford, G. S. (1992). Analogical reasoning and conceptual complexity in cognitive development. $\mathrm{Hu}$ man Development, 35, 193-218.

Holyoak, K. J., Junn, E. N., \& Billman, D. O. (1984). Development of analogical problem-solving skill. Child Development, 55, 2042-2055.

Inagaki, K. (1989). Developmental shift in biological inference processes: From similarity-based to category-based attribution. Human Development, 32, 79-87.

Inagaki, K. (1990). The effects of raising animals on children's biological knowledge. British Journal of Developmental Psychology, 8, 119-129.

Inagaki, K., \& Hatano, G. (1987). Young children's spontaneous personification as analogy. Child Development, $58,1013-1020$.

Kotovsky, L., \& Gentner, D. (1996). Comparison and categorization in the development of relational similarity. Child Development, 67, 2797-2822.

Kurtz, K. J., Miao, C., \& Gentner, D. (in press). Learning by analogical bootstrapping. Journal of the Learning Sciences.

Liben, L. S. (1999). Developing an understanding of external spatial representations. In I. E. Sigel (Ed.), The development of representational thought: Theoretical perspectives (pp. 297-321). Mahwah, $\mathrm{NJ}$ : Lawrence Erlbaum Associates, Inc.

Liben, L. S., \& Downs, R. M. (1989). Understanding maps as symbols: The development of map concepts in children. in H. W. Reese (Ed.), Advances in child development and behavior (Vol. 22, pp. 145-201). New York: Academic

Liben, L. S., \& Yekel, C. A. (1996). Preschoolers' understanding of plan and oblique maps: The role of geometric and representational correspondence. Child Development, 67, 2780-2796.

Lillard, A. S. (1993). Pretend play skills and the child's theory of mind. Child Development, 64, 348-371.

Loewenstein, J., \& Gentner, D. (1997, Aprii). Using comparisan to improve preschoolers' spatial mapping ability. Poster presented at the Biennial Meeting of the Society for Research in Child Development, Washington, DC.

Loewenstein, J., \& Gentner, D. (1998). Relational language facilitates analogy in children. Proceedings of the 20th Annual Conference of the Cognitive Science Society, 615-620.

Loewenstein, J., Thompson, L., \& Gentner, D. (1999). Analogical encoding facilitates knowledge transfer in negotiation. Psychonomic Bulletin \& Review, 6, 586-597.

Markman, A. B., \& Gentner, D. (1993). Structural alignment during similarity comparisons. Cognitive Psychology, 25, 431-467.

Markman, A. B., \& Gentner, D. (1997). The effects of alignability on memory storage. Psychological Science, 8, 363-367.

Marzolf, D. P., \& DeLoache, J. S. (1994). Transfer in young children's understanding of spatial representations. Child Development, 65, 1-15.

Marzolf, D. P., \& DeLoache, J. S. (1997). Search tasks as measures of cognitive development. In N. Foreman \& R. Gillett (Eds.), A handbook of spatial research paradigms and methodologies (Vol. 1, pp. 131-157). Hove, England: Psychology Press.

Marzolf, D. P., DeLoache, J. S., \& Kolstad, V. (1999). The role of relational similarity in young children's use of a scale model. Developmental Science, 2, 296-305. 
Mayer, R. E. (1992). Thinking, problem solving, cognition. San Francisco: Freeman.

Medin, D. L., Goldstone, R. L., \& Gentner, D. (1993). Respects for similarity. Psychological Review, $100,254-278$

Namy, L. L., \& Gentner, D. (in press). Making a silk purse out of two sow's ears: Young children's use of comparison in category leaming. Journal of Experimental Psychology: General.

Newcombe, N. S., \& Huttenlocher, J. (2000). Making space: The development of spatial representation and reasoning. Cambridge, MA: MIT Press.

Palincsar, A. S., \& Brown, A. L. (1984). Reciprocal teaching of comprehension fostering and comprehension-monitoring activities. Cognition and Instruction, 1, 117-175.

Perner, J. (1991). Understanding the representational mind. Cambridge, MA: MIT Press.

Plumert, J. M., Ewert, K., \& Spear, S. J. (1995). The early development of children's communication about nested spatial relations. Child Development, 66, 959-969.

Plumert, J. M., \& Nichols-Whitehead, P. (1996). Parental scaffolding of young children's spatial communication. Developmental Psychology, 32, 523-532.

Rattermann, M. J., \& Gentner, D. (2001). The effect of language on similarity: The use of relational labels improves young children's analogical mapping performance. Unpublished manuscript, Northwestern University.

Rattermann, M. J., \& Gentner, D. (1998a). The effect of language on similarity: The use of relational labels improves young children's performance in a mapping task. In K. Holyoak, D. Gentner, \& B. Kokinov (Eds.), Advances in analogyresearch: Integration of theory \& datafrom the cognitive, computational, and neural sciences (pp. 274-282). Sophia, Bulgaria: New Bulgarian University.

Rattermann, M. J., \& Gentner, D. (1998b). More evidence for a relational shift in the development of analogy: Children's performance on a causal-mapping task. Cognitive Development, 13, 453-478.

Reeves, L. M., \& Weisberg, R. W. (1994). The role of content and abstract information in analogical transfer. Psychological Bulletin, 115, 381-400.

Ross, B. H., \& Kennedy, P. T. (1990). Generalizing from the use of earlier examples in problem solving. Journal of Experimental Psychology: Learning, Memory, and Cognition, 16, 42-55.

Smith, L. B. (1984). Young children's understanding of attributes and dimensions: A comparison of conceptual and linguistic measures. Child Development, 55, 363-380.

Tan, L. S., \& Uttal, D. (1999, March). The development of the ability to impose a structure on a spatial pattern. Paper presented at the Biennial Meeting of the Society for Research in Child Development, Albuquerque, NM.

Thompson, L., Gentner, D., \& Loewenstein, J. (2000). Avoiding missed opportunities in managerial life: Analogical learning improves case-based transfer. Organizational Behavior and Human Decision Processes, 82, 60-75.

Uttal, D. H. (2000). Seeing the big picture: Map use and the development of spatial cognition. Developmental Science, 3, 247-286.

Uttal, D. H., Gregg, V. , Tan, L. S., Chamberlin, M., \& Sines, A. (2001). Connecting the dots: Children's use of a systematic figure to facilitate mapping and search. Developmental Psychology, 37, 1-13.

Uttal, D. H., Schreiber, J. C., \& DeLoache, J. S. (1995). Waiting to use a symbol: The effects of delay on children's use of models. Child Development, 66, 1875-1891.

Vosniadou, S. (1989). Analogical reasoning as a mechanism in knowledge acquisition: A developmental perspective. In S. Vosniadou \& A. Ortony (Eds.), Similarity and analogical reasoning (pp. 413 437). New York: Cambridge University Press.

Zacks, J., \& Tversky, B. (1999). Bars and lines: A study of graphic communication. Memory \& Cognition, 27, 1073-1079. 